\title{
Risk Factors of the Masticatory Function in Patients with Temporomandibular Disorders: A Cross-Sectional Cohort Study
}

\author{
Keon-Hyung Kim, Jo-Eun Park, Mee-Eun Kim, Hye-Kyoung Kim \\ Department of Oral Medicine, Collage of Dentistry, Dankook University, Cheonan, Korea
}

Received August 9, 2019

Revised August 21, 2019

Accepted August 21, 2019

\section{Correspondence to:}

Hye-Kyoung Kim

Department of Oral Medicine, Collage of

Dentistry, Dankook University, 119 Dandae-

ro, Dongnam-gu, Cheonan 31116, Korea

Tel: +82-41-550-1913

Fax: +82-505-434-7951

E-mail:12170144@dankook.ac.kr

https://orcid.org/0000-0002-0734-5533
Purpose: To investigate the masticatory function of patients with different temporomandibular disorders (TMD) phenotypes, and to explore the risk factors for the masticatory function of TMD patients among multiple biopsychosocial variables using patient-reported outcomes (PROs).

Methods: Clinical features and TMD diagnoses of 250 cases were investigated by reviewing medical records. Psychosocial factors were evaluated using four questionnaires representing pain severity and pain interference (Brief Pain Inventory), pain catastrophizing (Pain Catastrophizing Scale, PCS), psychological distress (Symptom Check List-90-Revised, SCL-90R) and kinesiophobia (Tampa Scale for Kinesiophobia for Temporomandibular Disorders, TSKTMD). Masticatory function, as a dependent variable, was determined using the Jaw Functional Limitation Scale (JFLS). Kruskal-Wallis test and Spearman's rank correlation were used for analyses.

Results: A total of 145 cases were included and classified into four subgroups including group 1: TMD with internal derangement without pain $(n=14)$, group 2: TMD with muscle pain $(n=32)$, group 3: TMD with joint pain $(n=60)$ and group 4: TMD with muscle-joint combined pain $(n=39)$. Pain severity $(p=0.001)$ and interference $(p=0.022)$ were the highest in group 2, but the mean global score of JFLS was the highest in group 3, followed by group 4, group 2, and group $1(\mathrm{p}=0.013)$. Pain severity, pain interference, the mean global score of PCS and the mean global score of TSK-TMD showed significant and moderate correlation with the mean global score of JFLS. All subdimensions and the global severity index of SCL-90R had significant, but weak correlations with all scores of JFLS.

Conclusions: The results suggest that masticatory functional limitation depends on the TMD phenotypes. Among the various PROs, pain perception, pain catastrophizing and kinesiophobia seem to be more influential risk factors on jaw function than psychological distress, such as depression and anxiety.

Key Words: Biopsychosocial; Catastrophization; Kinesiophobia; Masticatory function; Temporomandibular disorder

\section{INTRODUCTION}

Maintaining acceptable masticatory function is one of the main treatment goals of dentistry. Basal role of masticatory function, such as chewing and swallowing is the first step in the digestive process [1-3]. Emotional function such as laughing, screaming, and kissing is another dimension of masticatory function. Therefore, masticatory function is an impactful factor to a patient's general health condition and quality of life $[4,5]$. There are several factors influencing masticatory function, including the number of teeth, malocclusion, bite force, salivary function, and 
temporomandibular disorders (TMD) [1,3,6,7]. Among them, TMD is one of the major causes of masticatory functional limitation [5,7-9]. Furthermore, many studies showed relationship between TMD and masticatory function [8-11].

The findings of several existing literatures demonstrate that evaluation of masticatory function is an essential element in the diagnosis and treatment of TMD. In this context, measurement of functional limitation in TMD was included in Diagnostic Criteria for Temporomandibular Disorders (DC/TMD) [12]. Particularly, DC/TMD recommends Jaw Functional Limitation Scale (JFLS), a self-reported questionnaire, in the evaluation of masticatory function $[5,12]$.

To understand TMD as a major factor of masticatory functional limitation, the causes of TMD need to be investigated. Since an early and influential literature by Costen [13], the idea that malocclusion and related factors are fundamental and key causes of TMD was widely accepted. During decades, many dental researchers and clinicians had tried to explain TMD with a single factor such as joint, muscle, or occlusion, but the supporting evidence was sparsely found [14]. Recently, it is generally accepted that TMD is not caused by a single factor but a complex disorder with overlapping comorbidities of physical signs and symptoms [15]. With advances in neuroscience and cognitive science, concept of the biopsychosocial model for diagnosis and treatment of TMD has growing clinical and scientific support. Also, numerous studies and evidences show that multifactorial etiology is related to TMD and jaw function [16-20]. Several factors of the multifactorial etiology of TMD include biological factors, endogenous opioid function, differences in trauma, anatomical genotypes, parafunctions, occlusal changes, and psychosocial factors [12,16,20,21]. Besides to systemic and local factors, psychosocial factors such as pain coping, anxiety, depression, pain catastrophizing, and kinesiophobia have been recently emphasized [22-27]. Several psychosocial factors can predispose a person to TMD-pain, precipitate it, or prolong it [28].

Although the viewpoint of the pathophysiology of TMD has changed from occlusion to biopsychosocial model, there is still lack of research on effects of multidimensional biopsychosocial factors on the masticatory function of TMD patients. Therefore, the aims of the present study were (1) to investigate the masticatory function of patients with different TMD phenotypes using the JFLS questionnaire, and (2) to explore the risk factors for masticatory function of TMD patients among multiple biopsychosocial variables using patient-reported outcomes (PROs).

\section{MATERIALS AND METHODS}

\section{Subjects}

Subjects were recruited from the patients who visited the Orofacial Pain Clinic of Dankook University Dental Hospital, Cheonan, Korea for a complaint of TMD including jaw pain, mouth opening limitation and joint noise from January to February 2019. Inclusion criteria were those who were diagnosed as primary TMD; patients who fully completed the questionnaires; patients aged 18 or older. Exclusion criteria were as follows: (1) Patients who refused to use their data for research; (2) Patients with a history of trauma or recent surgery or fibromyalgia; (3) Patients who had oral disorder affecting masticatory function (e.g., oral ulcer, tooth pain); (4) Patients under dental treatment; (5) Patients wearing a denture or lack of posterior occlusion; (6) Patients with systemic disease classified as American Society of Anesthesiologists Physical Status classification 3 or more [29]; (7) Patient with impaired salivary function affecting masticatory function. This study was approved by Institutional Review Board committee of Dankook University Dental Hospital (IRB no. DKUDH IRB 2019-01-004).

\section{Data Collection}

All data, including sex, age, pain duration, number of teeth, were collected retrospectively using the medical records. All patients were asked to complete the questionnaires in the waiting room before the consultation. Informed consent for using their clinical data for the research purpose was obtained. TMD examination was conducted according to the Axis I of the Research Diagnostic Criteria for Temporomandibular Disorders (RDC/TMD) [30] by one of five experts (K.M.E; K.H.K; P.J.E; K.K.H; J.K.H). Then, classification into clinically distinct four subgroups was done for further statistical analysis, i.e., (1) group 1: TMD with internal derangement without pain (TMD_ID, 
$\mathrm{n}=14$ ), (2) group 2: TMD with muscle pain (TMD_M, $\mathrm{n}=32$ ), (3) group 3: TMD with joint pain (TMD_J, $n=60$ ) and (4) group 4: TMD with muscle-joint combined pain (TMD_MJ, $n=39)$.

\section{Questionnaires}

PROs representing the pain perception, pain catastrophizing, psychological distress and kinesiophobia were measured by four questionnaires. Masticatory function, as a dependent variable, was measured with the JFLS. Therefore, a total of five questionnaires were used in this study.

\section{1) Jaw Functional Limitation Scale}

The JFLS is an organ-specific instrument for assessing overall functional limitation of the masticatory system comprising three constructs including mastication, vertical jaw mobility and emotional and verbal expression. JFLS yields a global score from a total of 20 items and exhibits excellent psychometric properties respect to internal consistency, reliability and validity [5,31]. All items presented with 0-10 numeric scales. In this study, the Korean version of JFLS (JFLS-K) was used [32]. Internal consistencies of three subscales of JFLS-K were 0.88 (mastication), 0.79 (vertical jaw mobility) and 0.95 (verbal and emotional expression). Testretest reliability for mastication, vertical jaw mobility, and verbal and emotional expression was 0.86, 0.84, and 081, respectively.

\section{2) Brief Pain Inventory}

The Brief Pain Inventory (BPI) is a short, simple, self-administered questionnaire to assess the severity of pain and the impact of pain on daily functions. It has been adopted worldwide for clinical pain assessment. It contains 11-items presented with 0-10 numeric scales. BPI pain severity includes four items: worst; least; average; now. BPI pain interference items include seven items: general activity; mood; walking ability; normal work (including housework); relations with other people; sleep; enjoyment of life [33,34]. In this study, the Korean version of BPI [35] was used. The item related to walking ability was replaced by one related to chewing ability considering the different pain profiles of TMD. Responses were based on a week before the first visit.

\section{3) Pain Catastrophizing Scale}

The Pain Catastrophizing Scale (PCS) developed by Sullivan et al. [36] is one of the most widely used instruments for measuring catastrophic thinking related to pain. It contains 13-items on a 5-point scale with 0 "not at all" to 4 "all the time". This 13-items includes six items for assessing helplessness, four items for assessing rumination, and three items for assessing magnification. The PCS yields a total score and three subscale scores [37,38].

\section{4) Symptom Check List-90-Revised}

The Symptom Checklist-90-Revised (SCL-90R) is a method to evaluate psychological problems and identify symptoms [39]. Participants are required to respond to the 90-items using a 5-point rating scale with 0 "not at all" to 4 "extremely". It evaluates nine symptom dimensions (somatization, obsessive-compulsive, interpersonal sensitivity, depression, anxiety, hostility, phobic anxiety, paranoid ideation, and psychoticism) and three global distress indices (global severity index [GSI], positive symptom distress index, and positive symptom total).

5) Tampa Scale for Kinesiophobia for Temporomandibular Disorders

The Tampa Scale for Kinesiophobia for Temporomandibular Disorders (TSK-TMD) is an adaptive version of the TSK-TMD patients. TSK assesses fear of movement which considered as a strong predictor of disability in patients with musculoskeletal disorders, such as low back pain, fibromyalgia, and osteoarthritis. Global scale known as the fear of movement consists of two subscales, activity avoidance and somatic focus. TSK-TMD has good reliability (0.83) and satisfactory validity (0.23) [27]. In this study, Korean version of TSK-TMD [40] was used. It includes 12-items using a 4-point rating scale with 1 "strongly disagree" to 4 "strongly agree".

\section{Statistical Analysis}

The descriptive data for continuous variables were represented as mean and standard deviation. The comparison of quantitative data from demographics and self-reported questionnaires between TMD subgroups were performed by Kruskal-Wallis test. For categorical variables, the chi-square 
test was used. Spearman's rank correlation was used to assess the association between JFLS-K and demographics (age, sex, and pain duration), four TMD subgroups, TMD pain (pain severity and pain interference from BPI), psychological distress (9 subscales and global score from SCL90R), pain catastrophizing (3 subscales and global score from PCS) and kinesiophobia (2 subscales and global score). Statistical analyses were conducted using IBM SPSS Statistics for Windows, Version 21.0 (IBM Co., Armonk, NY, USA) and two-tailed level of statistical significance was set at 0.05 .

\section{RESULTS}

\section{Subjects Characteristics}

As shown in a sample flow chart in Fig. 1, a total of 250 patients visited the hospital for TMD symptoms during given period. Among them, 54 patients were excluded since not diagnosed yet. According to the inclusion and exclusion criteria, 51 patients were further excluded. They were as follows: patients aged under $18(n=18)$, patients with uncompleted questionnaires $(n=22)$, patients diagnosed as secondary TMD $(n=2)$, patients under orthodontic treatment $(n=5)$, patients wearing denture $(n=2)$, and patients with

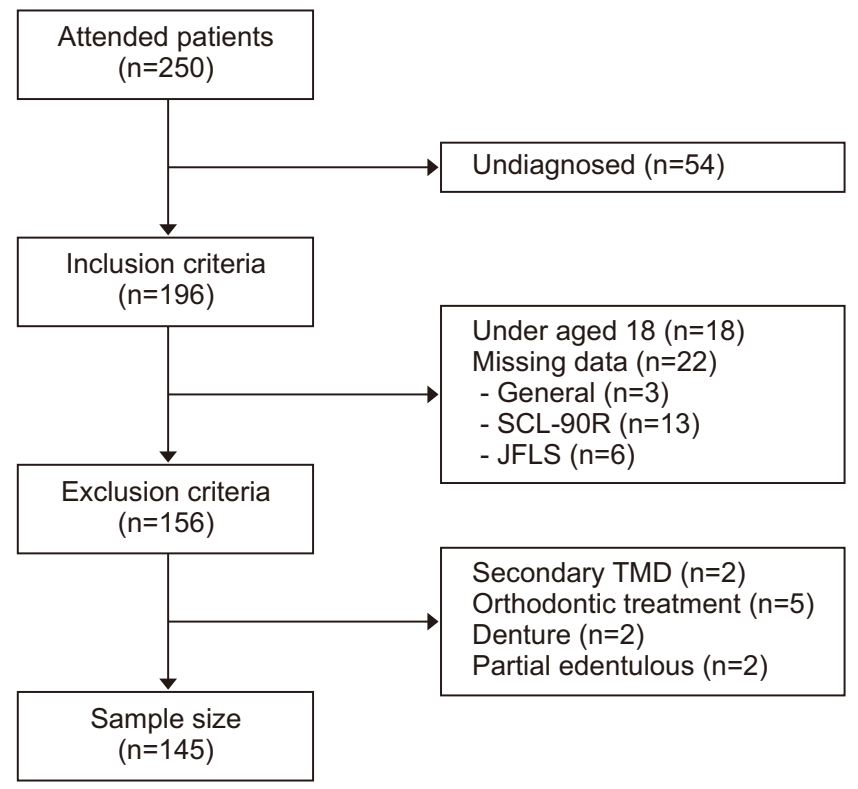

Fig. 1. Study sample flow chart. SCL-90R, Symptom Check List 90-revised; JFLS, Jaw Functional Limitation Scale; TMD, temporomandibular disorder. edentulous ridge on one side $(n=2)$. Therefore, a total of 145 subjects were included in this study.

\section{Demographic Characteristics, TMD Pain, Pain Catastrophizing and Psychological Distress of Four TMD Subgroups}

Table 1 presents the demographic characteristics (age, sex, pain duration, and the number of teeth), pain perception (pain severity and pain interference), pain catastrophizing and psychological distress according to four TMD subgroups. The proportion of patients was the highest in TMD_J (41.4\%), followed by TMD_MJ (26.9\%), TMD_M (22.1\%), and TMD_ID (9.7\%). The mean age of TMD_M was the highest, followed by TMD_MJ, TMD_J and TMD_ID $(P=0.003)$. Sex $(p=0.797)$ and the number of teeth $(p=0.687)$ didn't exhibit statistical differences between the four subgroups. TMD_MJ showed the longest pain duration and followed by TMD_M and TMD_J had the shortest pain duration ( $p=0.047)$. Among the four groups, the mean scores of pain severity $(p=0.001)$ and pain interference $(p=0.022)$ were the highest in TMD_M while they were the lowest in TMD_ J. In the comparison of PCS scores among the four TMD subgroups, TMD_M showed the highest mean global score of PCS among the all groups, but it did not reach statistical significance. Among the nine subdimensions and GSI of SCL-90R, all subdimensions except phobic anxiety showed group difference. The score of all subdimensions except phobic anxiety was higher in the order of TMD_M, TMD_ MJ, TMD_J and TMD_ID (all p<0.05), i.e., TMD_M exhibited the highest score in the somatization, obsessive-compulsive, interpersonal sensitivity, depression, anxiety, hostility, paranoid ideation, psychoticism, and GSI.

\section{TSK-TMD and JFLS of Four TMD Subgroups}

The mean scores and standard deviations of the TSKTMD and JFLS according to four TMD groups were presented in Table 2. In TSK-TMD, two subdimensions of activity avoidance $(p=0.538)$ and somatic focus $(p=0.942)$ and global score $(p=0.894)$ did not show statistical difference between four groups. On the other hand, the mean mastication score among the three subcategories of JFLS was the highest in TMD_J, followed by TMD_MJ, and TMD_M, TMD_ID ( $\mathrm{p}=0.001)$. Mobility $(\mathrm{p}=0.078)$ and communication 
Table 1. Demographics, TMD pain, pain catastrophizing and psychological distress according to four different TMD subgroups ( $n=145$ )

\begin{tabular}{|c|c|c|c|c|c|c|c|}
\hline Characteristics & TMD_ID (1) & TMD_M (2) & TMD_J (3) & TMD_MJ (4) & $\chi^{2}$ & p-value & Rank \\
\hline \multicolumn{8}{|l|}{ Demographics } \\
\hline Patients number (\%) & $14(9.7)$ & $32(22.1)$ & $60(41.4)$ & $39(26.9)$ & & & \\
\hline Age $(y)$ & $30.0 \pm 13.6$ & $45.5 \pm 15.9$ & $34.3 \pm 15.0$ & $36.5 \pm 16.1$ & 13.647 & $0.003^{*}$ & $2>4>3>1$ \\
\hline Sex, female & 8 & 23 & 39 & 26 & 1.016 & 0.797 & \\
\hline Pain duration (mo) & $43.2 \pm 64.1$ & $24.8 \pm 44.5$ & $10.7 \pm 22.1$ & $31.2 \pm 64.8$ & 7.935 & $0.047 *$ & $4>2>3$ \\
\hline The number of teeth & $27.5 \pm 1.1$ & $27.4 \pm 1.1$ & $27.3 \pm 1.6$ & $27.6 \pm 0.9$ & 1.478 & 0.687 & \\
\hline \multicolumn{8}{|l|}{ BPI } \\
\hline Pain severity & $1.1 \pm 1.3$ & $3.4 \pm 2.2$ & $3.1 \pm 1.9$ & $3.2 \pm 1.8$ & 17.479 & $0.001 *$ & $2>4>3$ \\
\hline Pain interference & $1.2 \pm 1.8$ & $3.3 \pm 2.8$ & $2.6 \pm 2.1$ & $2.8 \pm 2.4$ & 9.625 & $0.022^{*}$ & $2>4>3$ \\
\hline \multicolumn{8}{|l|}{ PCS } \\
\hline Magnification & $3.0 \pm 3.4$ & $4.8 \pm 3.5$ & $3.9 \pm 2.8$ & $4.4 \pm 4.9$ & 3.865 & 0.276 & \\
\hline Rumination & $3.0 \pm 3.3$ & $5.6 \pm 5.0$ & $3.8 \pm 3.9$ & $3.7 \pm 3.6$ & 3.772 & 0.287 & \\
\hline Helplessness & $3.7 \pm 4.5$ & $6.9 \pm 5.9$ & $6.0 \pm 4.6$ & $5.4 \pm 4.7$ & 5.489 & 0.139 & \\
\hline Global score & $9.7 \pm 10.9$ & $17.4 \pm 13.9$ & $13.8 \pm 10.4$ & $13.7 \pm 11.8$ & 5.181 & 0.159 & \\
\hline \multicolumn{8}{|l|}{ SCL-90R } \\
\hline Somatization & $42.1 \pm 6.6$ & $51.6 \pm 9.4$ & $44.4 \pm 5.4$ & $48.5 \pm 12.9$ & 19.205 & $<0.001 *$ & $2>4>3>1$ \\
\hline Obsessive-compulsive & $38.0 \pm 7.6$ & $46.9 \pm 9.2$ & $41.1 \pm 7.4$ & $45.2 \pm 12.8$ & 12.230 & $0.007 *$ & $2>4>3>1$ \\
\hline Interpersonal sensitivity & $39.6 \pm 7.8$ & $46.9 \pm 9.5$ & $41.7 \pm 7.3$ & $44.6 \pm 12.5$ & 10.768 & $0.013^{*}$ & $2>4>3>1$ \\
\hline Depression & $38.2 \pm 4.9$ & $47.2 \pm 10.5$ & $40.5 \pm 6.1$ & $45.1 \pm 12.1$ & 14.498 & $0.002^{*}$ & $2>4>3>1$ \\
\hline Anxiety & $40.0 \pm 5.6$ & $48.5 \pm 12.1$ & $41.6 \pm 5.4$ & $45.0 \pm 12.3$ & 13.297 & $0.004^{*}$ & $2>4>3>1$ \\
\hline Hostility & $41.3 \pm 3.4$ & $47.3 \pm 8.0$ & $43.0 \pm 5.9$ & $45.4 \pm 11.2$ & 9.100 & $0.028^{*}$ & $2>4>3>1$ \\
\hline Phobic anxiety & $42.0 \pm 2.3$ & $44.7 \pm 4.7$ & $43.7 \pm 4.3$ & $46.8 \pm 13.9$ & 3.532 & 0.317 & \\
\hline Paranoid & $39.3 \pm 2.6$ & $46.6 \pm 9.9$ & $41.1 \pm 5.9$ & $44.2 \pm 12.2$ & 14.750 & $0.002^{*}$ & $2>4>3>1$ \\
\hline Psychosis & $40.0 \pm 3.3$ & $48.3 \pm 11.6$ & $41.3 \pm 5.5$ & $45.0 \pm 12.2$ & 12.806 & $0.005^{*}$ & $2>4>3>1$ \\
\hline GSI & $37.2 \pm 5.4$ & $47.3 \pm 10.4$ & $40.0 \pm 6.2$ & $44.4 \pm 13.6$ & 16.664 & $0.001 *$ & $2>4>3>1$ \\
\hline
\end{tabular}

TMD, temporomandibular disorder; TMD_ID (1), TMD with internal derangement without pain; TMD_M (2), TMD with muscle pain; TMD_J (3), TMD with joint pain; TMD_MJ (4), TMD with muscle-joint combined pain; BPI, Brief Pain Inventory; PCS, Pain Catastrophizing Scale; SCL-90R, Symptom Check List 90-revised; GSI, Global Severity Index from SCL-90R.

Values are presented as number (\%), mean \pm standard deviation, or number only.

$\mathrm{p}$-values were obtained from Kruskal-Wallis test.

${ }^{*} \mathrm{p}<0.05$.

Table 2. TSK-TMD and Jaw Function Limitation Scale according to four different TMD subgroups

\begin{tabular}{|c|c|c|c|c|c|c|c|}
\hline Questionnaires & TMD_ID (1) & TMD_M (2) & TMD_J (3) & TMD_MJ (4) & $\chi^{2}$ & $p$-value & Rank \\
\hline \multicolumn{8}{|l|}{ TSK } \\
\hline Activity avoidance & $14.5 \pm 4.5$ & $14.8 \pm 5.2$ & $15.6 \pm 4.4$ & $16.2 \pm 4.4$ & 2.169 & 0.538 & \\
\hline Somatic focus & $9.8 \pm 2.5$ & $10.25 \pm 3.8$ & $9.6 \pm 2.8$ & $9.9 \pm 3.0$ & 0.394 & 0.942 & \\
\hline Global score & $24.3 \pm 6.5$ & $25.0 \pm 8.4$ & $25.3 \pm 6.5$ & $26.2 \pm 6.9$ & 0.612 & 0.894 & \\
\hline \multicolumn{8}{|c|}{ Jaw Function Limitation Scale } \\
\hline Mastication & $0.8 \pm 0.9$ & $2.7 \pm 2.3$ & $3.1 \pm 2.0$ & $2.9 \pm 1.8$ & 16.561 & $0.001 *$ & $3>4>2>1$ \\
\hline Mobility & $1.4 \pm 1.4$ & $2.6 \pm 2.3$ & $2.8 \pm 1.8$ & $2.5 \pm 1.7$ & 6.816 & 0.078 & \\
\hline Communication & $0.2 \pm 0.6$ & $1.3 \pm 2.2$ & $0.8 \pm 1.4$ & $1.1 \pm 1.6$ & 4.271 & 0.234 & \\
\hline Global score & $0.9 \pm 0.7$ & $2.0 \pm 1.8$ & $2.2 \pm 1.3$ & $2.1 \pm 1.5$ & 10.704 & $0.013^{*}$ & $3>4>2>1$ \\
\hline
\end{tabular}

TSK-TMD, Tampa Scale for Kinesiophobia for Temporomandibular Disorders; TMD, temporomandibular disorder; TMD_ID (1), TMD with internal derangement without pain; TMD_M (2), TMD with muscle pain; TMD_J (3), TMD with joint pain; TMD_MJ (4), TMD with muscle-joint combined pain.

Values are presented as mean and standard deviation.

$p$-values were obtained from the Kruskal-Wallis test.

$*_{p}<0.05$. 
( $p=0.234)$ scores did not present the statistical difference between groups. The mean global score of JFLS, like the mean mastication score, was the highest in TMD_J, followed by TMD_MJ, and TMD_M, TMD_ID ( $\mathrm{p}=0.013)$.

\section{Correlations between JFLS and TMD Pain, Pain Catastrophizing, Psychological Distress and Kinesiophobia}

There were no significant correlations between JFLS and sex $(p=0.226)$, age $(p=0.070)$, pain duration $(p=0.111)$ and

Table 3. Correlations between Jaw Function Limitation Scale and TMD pain

\begin{tabular}{ccc}
\hline \multirow{2}{*}{ Spearman's rank correlation } & \multicolumn{2}{c}{ Brief Pain Inventory } \\
\cline { 2 - 3 } & Pain severity & Pain interference \\
\hline JFLS_mastication & & \\
Coefficient & $0.509^{\star *}$ & $0.531^{\star *}$ \\
p-value & $<0.001$ & $<0.001$ \\
JFLS_mobility & & \\
Coefficient & $0.483^{* *}$ & $0.490^{\star *}$ \\
p-value & $<0.001$ & $<0.001$ \\
JFLS_communication & & \\
Coefficient & $0.460^{\star *}$ & $0.564^{\star *}$ \\
p-value & $<0.001$ & $<0.001$ \\
JFLS_global score & & \\
Coefficient & $0.608^{\star *}$ & $0.632^{\star *}$ \\
p-value & $<0.001$ & $<0.001$ \\
\hline
\end{tabular}

JFLS, Jaw Functional Limitation Scale.

$\mathrm{p}$-values were obtained from the Spearman's rank correlation. ${ }^{* *} p<0.001$. the number of teeth $(\mathrm{p}=0.186)$ (data are not shown). Table 3 exhibits correlations between JFLS and TMD pain from BPI. Both pain severity $(r=0.608, p<0.001)$ and pain interference $(r=0.632, p<0.001)$ showed significant and moderate correlations with the mean global score of JFLS. In the comparison of three subcategories of JFLS with TMD pain, mastication, mobility, and communication also presented significant correlations with pain severity $(r=0.509, r=483$, $\mathrm{r}=0.460$, respectively; all $\mathrm{p}<0.001$ ) and pain interference $(\mathrm{r}=0.531, \mathrm{r}=0.490, \mathrm{r}=0.564$, respectively; all $\mathrm{p}<0.001)$. Table 4 presents the correlations between JFLS and PCS. The mean global score of PCS showed significant and moderate correlation with the mean global score of JFLS ( $r=0.531$, $\mathrm{p}<0.001)$. Three components of PCS including magnification, rumination, and helplessness also showed significant correlations with all dimensions of JFLS (all $\mathrm{p}<0.001$ ). Nine dimensions and GSI of SCL-90R showed significant and mild correlations with the mean global score of JFLS, i.e., somatization $(r=0.295, p<0.001)$, obsessive-compulsive $(r=0.251, p=0.002)$, interpersonal sensitivity $(r=0.180$, $\mathrm{p}=0.031)$, depression $(\mathrm{r}=0.216, \mathrm{p}=0.009)$, anxiety $(\mathrm{r}=0.297$, $\mathrm{p}<0.001)$, hostility $(\mathrm{r}=0.197, \mathrm{p}=0.017)$, paranoid ideation $(\mathrm{r}=0.301, \mathrm{p}<0.001)$, psychoticism $(\mathrm{r}=0.336, \mathrm{p}<0.001)$ and GSI $(\mathrm{r}=0.295, \mathrm{p}<0.001)$. All three components of JFLS including mastication, mobility, and communication also showed significant and mild correlations with all dimensions of SCL90R (except between mobility and hostility) (Table 5). Table

Table 4. Correlations between Jaw Function Limitation Scale and Pain Catastrophizing Scale

\begin{tabular}{|c|c|c|c|c|}
\hline \multirow{2}{*}{ Spearman's rank correlation } & \multicolumn{4}{|c|}{ Pain Catastrophizing Scale } \\
\hline & Magnification & Rumination & Helplessness & Global score \\
\hline \multicolumn{5}{|l|}{ JFLS_mastication } \\
\hline Coefficient & $0.395^{\star \star}$ & $0.442^{* \star}$ & $0.491 * \star$ & $0.478^{\star \star}$ \\
\hline$p$-value & $<0.001$ & $<0.001$ & $<0.001$ & $<0.001$ \\
\hline \multicolumn{5}{|l|}{ JFLS_mobility } \\
\hline Coefficient & $0.401 * *$ & $0.378^{* *}$ & $0.431 * *$ & $0.419 * *$ \\
\hline$p$-value & $<0.001$ & $<0.001$ & $<0.001$ & $<0.001$ \\
\hline \multicolumn{5}{|l|}{ JFLS_communication } \\
\hline Coefficient & $0.413^{* *}$ & $0.442^{* *}$ & $0.482^{* *}$ & $0.472 * *$ \\
\hline $\mathrm{p}$-value & $<0.001$ & $<0.001$ & $<0.001$ & $<0.001$ \\
\hline \multicolumn{5}{|l|}{ JFLS_ global score } \\
\hline Coefficient & $0.447 * \star$ & $0.492^{* *}$ & $0.553^{* *}$ & 0.531 ** \\
\hline p-value & $<0.001$ & $<0.001$ & $<0.001$ & $<0.001$ \\
\hline
\end{tabular}

JFLS, Jaw Functional Limitation Scale.

p-values were obtained from the Spearman's rank correlation.

${ }^{* *} p<0.001$. 
Table 5. Correlations between Jaw Function Limitation Scale and SCL-90R

\begin{tabular}{|c|c|c|c|c|c|c|c|c|c|c|}
\hline \multirow{2}{*}{$\begin{array}{l}\text { Spearman's rank } \\
\text { correlation }\end{array}$} & \multicolumn{10}{|c|}{ SCL-90R } \\
\hline & Som & $\mathrm{O}-\mathrm{C}$ & I-S & Dep & Anx & Hos & $P-A$ & Par & Psy & GSI \\
\hline \multicolumn{11}{|l|}{ JFLS_mastication } \\
\hline Coefficient & $0.252^{*}$ & $0.234^{*}$ & $0.236^{*}$ & $0.220^{*}$ & $0.316^{* *}$ & $0.218^{*}$ & $0.281 *$ & $0.197 *$ & $0.328^{* *}$ & $0.301 * *$ \\
\hline$p$-value & 0.002 & 0.005 & 0.004 & 0.008 & $<0.001$ & 0.008 & 0.001 & 0.001 & $<0.001$ & $<0.001$ \\
\hline \multicolumn{11}{|l|}{ JFLS_mobility } \\
\hline Coefficient & $0.180^{*}$ & $0.220^{*}$ & $0.166^{*}$ & $0.183^{\star}$ & $0.207^{*}$ & 0.137 & $0.223^{*}$ & $0.207^{*}$ & $0.199 *$ & $0.214 *$ \\
\hline$p$-value & 0.031 & 0.008 & 0.045 & 0.028 & 0.012 & 0.100 & 0.007 & 0.012 & 0.016 & 0.010 \\
\hline \multicolumn{11}{|l|}{ JFLS_communication } \\
\hline Coefficient & $0.367^{\star \star}$ & $0.282^{*}$ & $0.206^{*}$ & $0.289 * *$ & $0.346^{\star \star}$ & $0.206^{*}$ & $0.380 * \star$ & $0.244^{*}$ & $0.380 * *$ & 0.350 ** \\
\hline p-value & $<0.001$ & 0.001 & 0.013 & $<0.001$ & $<0.001$ & 0.013 & $<0.001$ & 0.003 & $<0.001$ & $<0.001$ \\
\hline \multicolumn{11}{|l|}{ JFLS_global score } \\
\hline Coefficient & $0.295^{\star \star}$ & $0.251^{*}$ & $0.180 *$ & $0.216^{*}$ & $0.297 * \star$ & $0.197 *$ & $0.301 * *$ & $0.207^{\star}$ & $0.335^{* *}$ & $0.295^{\star *}$ \\
\hline$p$-value & $<0.001$ & 0.002 & 0.031 & 0.009 & $<0.001$ & 0.017 & $<0.001$ & 0.013 & $<0.001$ & $<0.001$ \\
\hline
\end{tabular}

SCL-90R, Symptom Check List 90-revised; Som, somatization; O-C, obsessive-compulsive; I-S, interpersonal sensitivity; Dep, depression; Anx, anxiety; Hos, hostility; P-A, phobic anxiety; PAR, paranoid ideation; PSY, psychoticism; GSI, Global Severity Index from SCL-90R; JFLS, Jaw Functional Limitation Scale.

$\mathrm{p}$-values were obtained from the Spearman's rank correlation.

${ }^{*} p<0.05,{ }^{* *} p<0.001$.

Table 6. Correlations between Jaw Function Limitation Scale and Tampa Scale for Kinesiophobia

\begin{tabular}{|c|c|c|c|}
\hline \multirow{2}{*}{$\begin{array}{l}\text { Spearman's rank } \\
\text { correlation }\end{array}$} & \multicolumn{3}{|c|}{ Tampa Scale for Kinesiophobia } \\
\hline & $\begin{array}{c}\text { Activity } \\
\text { avoidance }\end{array}$ & $\begin{array}{l}\text { Somatic } \\
\text { focus }\end{array}$ & $\begin{array}{l}\text { Global } \\
\text { score }\end{array}$ \\
\hline \multicolumn{4}{|l|}{ JFLS_mastication } \\
\hline Coefficient & $0.373^{* *}$ & $0.378^{* *}$ & $0.408^{* *}$ \\
\hline$p$-value & $<0.001$ & $<0.001$ & $<0.001$ \\
\hline \multicolumn{4}{|l|}{ JFLS_mobility } \\
\hline Coefficient & $0.507^{\star *}$ & $0.407^{* *}$ & $0.515^{\star *}$ \\
\hline$p$-value & $<0.001$ & $<0.001$ & $<0.001$ \\
\hline \multicolumn{4}{|c|}{ JFLS_communication } \\
\hline Coefficient & $0.301 * *$ & $0.391^{* *}$ & $0.366^{\star *}$ \\
\hline$p$-value & $<0.001$ & $<0.001$ & $<0.001$ \\
\hline \multicolumn{4}{|l|}{ JFLS_global score } \\
\hline Coefficient & $0.414^{* *}$ & $0.433^{* *}$ & $0.461 * *$ \\
\hline$p$-value & $<0.001$ & $<0.001$ & $<0.001$ \\
\hline
\end{tabular}

JFLS, Jaw Functional Limitation Scale.

$\mathrm{p}$-values were obtained from the Spearman's rank correlation.

${ }^{* *} p<0.001$.

6 indicates correlations between JFLS and TSK-TMD. The mean global score of JFLS showed significant and moderate correlation with the global score of TSK-TMD ( $\mathrm{r}=0.461$, $\mathrm{p}<0.001)$. Activity avoidance $(\mathrm{r}=0.414, \mathrm{p}<0.001)$ and somatic focus $(r=0.433, p<0.001)$, two dimensions of the TSKTMD, also presented significant correlations with JFLS, respectively.

\section{DISCUSSION}

The present study evaluated the masticatory function of TMD patients with four different phenotypes using the JFLS questionnaire, and found that mechanical jaw function was different according to the TMD subtypes. TMD_J showed the highest masticatory functional limitation, followed by TMD_MJ, TMD_M and TMD_ID. Among multiple biopsychosocial variables as the potential risk factors for the masticatory function using PROs, pain perception (pain severity and pain interference from BPI), pain catastrophizing and kinesiophobia showed significant and moderate correlations with the global score of JFLS. On the other hand, the global score of JFLS exhibited weak correlations with most subdimensions of SCL-90R.

The mean scores of pain severity and interference and all dimensions except phobic anxiety of SCL-90R were the highest in TMD_M, followed by TMD_MJ and TMD_J (Table 1). Liu and Steinkeler [41] said that more than 50\% of TMD are myofascial pain and TMD_M and TMD_MJ in this study probably contain patients with myofascial pain mostly. Previous studies presented that myofascial TMD patients are associated with extensive central sensitization and thereby have high pain intensity and pain perception $[42,43]$. Therefore, it is not surprising that two muscle pain groups show the higher mean scores of pain perception. Also, the 
order between groups in the all subdimensions of SCL-90R can be acceptable. According to Healy et al. [44], treatment for chronic myofascial pain patients could be affected by psychological characteristics.

Two subdimensions and the global score of TSK-TMD didn't show statistical difference among the four TMD subgroups (Table 2). Gil-Martínez et al. [20] showed that kinesiophobia is a predictor for pain and disability of TMD patients and thereby has important clinical significance of assessing and treating TMD. In terms of kinesiophobia, pain catastrophizing is advocated to predispose patients toward the development of kinesiophobia which may lead to aggravated disability and an enhancing of the overall pain experience [45]. In this study, the scores of pain catastrophizing did not show statistical significance between groups. In this context, it is not surprising that the mean scores of kinesiophobia had no significant difference between groups. In addition, Gil-Martínez et al. [46] showed the same result that no differences of kinesiophobia were found between muscle, joint and combined groups.

Unlike pain catastrophizing and kinesiophobia, the global score of JFLS, particularly mastication dimension, was the highest in TMD_J, followed by TMD_MJ, TMD_M, and TMD_ID (Table 2). This finding suggests that masticatory function depends on the TMD subtypes. Although pain experience and psychological distress were higher in TMD_M than in TMD_J, interestingly, subjective perception of masticatory functional limitation was higher in TMD_J than in TMD_M. This result also suggests that masticatory functional limitation, especially the mastication subscale, may be more related to mechanical or structural jaw dysfunction resulting from joint pain than dysfunctional muscle pain with increased pain intensity and psychological distress. Further studies are needed to validate of this result in a large sample deserves.

In Table 3, both pain severity and pain interference showed significant and moderate correlations with the global score and all three subscales of JFLS. This finding is consistent with previous studies indicating that TMD, especially TMDpain is one of the major causes of masticatory functional limitation. Among the three subscales, it is not surprising that mastication and mobility are correlated with pain severity and pain interference, since it is well known that provocation, such as chewing or yawning aggravates the TMD-pain [10].

Like TMD pain, three subdimensions and the global score of PCS showed significant and moderate correlations with masticatory functional limitation (Table 4). Pain catastrophizing, a magnified negative tendency during actual or anticipated unpleasant stimulation, has been recently emphasized as one of the contributors for TMD development $[15,26]$. Interestingly, the result of this study suggests that pain catastrophizing may have negative effect on masticatory function as well as pain experience of patients with TMD.

Many previous researches revealed that psychologic distress, such as somatization, depression and anxiety is one of the risk factors to painful TMD [15,17,44,47]. This study found that all subdimensions and the GSI of SCL90R have significant, but weak correlations with all scores of JFLS (except between mobility and hostility) (Table 5). Consistent with the results of the present study, Ohrbach et al. [31] demonstrated that comparison of global jaw limitation scores to depression, somatization and anxiety scores presented poor correlation. These results indicate that pain catastrophizing may have higher impact to masticatory function than psychological distress.

Kinesiophobia was emphasized in recent studies as a new rising predictor of pain and disability in TMD patients [27,40,46,48]. Gil-Martínez et al. [46] suggested that kinesiophobia should be included in the psychosocial evaluation and treatment of TMD patients. Since Visscher et al. [27] adopted the TSK to use for TMD patients, appropriate versions for different languages and cultures of TSK-TMD were applied $[40,48]$. In the current study, the mean scores of TSK-TMD showed significant and moderate correlation with all scores of JFLS (Table 6). According to the above result, this study can highly suggest that kinesiophobia might be one of the important risk factors for masticatory function in TMD patients.

The strengths of this study should be mentioned. It was not easy to compare to the results of this study with other relevant literature because the influence of the multidimensional biopsychosocial factors on jaw function using the JFLS questionnaire has not been studied. In this study, JFLS, an organ-specific valid instrument, was used to assess 
overall functional limitation of the masticatory system. JFLS is comprised of three constructs including mastication, vertical jaw mobility and emotional and verbal expression and exhibits excellent psychometric properties [5]. This study explored the multidimensional risk factors on the masticatory function using the validated questionnaires representing PROs in TMD patients with four different subtypes.

However, this study lacks of objective and quantitative measurement methods to evaluate masticatory ability. Several objective measurement methods have been developed and to evaluate masticatory performance. Sieving of chewed natural or artificial food and following determination of the mean particle size is still thought to be the "gold standard" for the quantification of masticatory efficiency [49]. One of the other objective methods to assess the chewing process is surface electromyography but the results of the studies still have been controversial [50]. Meanwhile, Boretti et al. [1] mentioned that masticatory function refers to a patients' subjective response about chewing and their objective capacity to chew. It deserves further research on the effects of biopsychosocial factors on masticatory function using objective assessment. Another shortcoming of this study is limited sample size. In order to follow inclusion and exclusion criteria, a large number of patients were excluded. The reproducibility of this study needs to be validated in a large sample.

In conclusion, this cross-sectional study found that the global score of JFLS was the highest in patients with TMD with joint pain, followed by TMD with muscle-joint combined pain, muscle pain and TMD with internal derangement without pain. This suggests that masticatory functional limitation, particularly mastication component, depends on the TMD phenotypes. Among the various PROs, pain perception, pain catastrophizing and kinesiophobia seem to be more influential risk factors on jaw function than psychological distress including depression and anxiety. This also suggests that treatments focusing on not only reduction of pain severity but also an approach to reduce pain catastrophizing and kinesiophobia are should be considered in order to improve masticatory function in patients with TMD.

\section{CONFLICT OF INTEREST}

No potential conflict of interest relevant to this article was reported.

\section{ORCID}

\author{
Keon-Hyung Kim \\ https://orcid.org/0000-0001-9850-8723 \\ Jo-Eun Park \\ https://orcid.org/0000-0003-0190-9972 \\ Mee-Eun Kim \\ https://orcid.org/0000-0001-9332-532X \\ Hye-Kyoung Kim \\ https://orcid.org/0000-0002-0734-5533
}

\section{REFERENCES}

1. Boretti G, Bickel M, Geering AH. A review of masticatory ability and efficiency. J Prosthet Dent 1995;74:400-403.

2. Nakata M. Masticatory function and its effects on general health. Int Dent J 1998;48:540-548.

3. Magalhães IB, Pereira LJ, Marques LS, Gameiro GH. The influence of malocclusion on masticatory performance. A systematic review. Angle Orthod 2010;80:981-987.

4. Reisine ST, Fertig J, Weber J, Leder S. Impact of dental conditions on patients' quality of life. Community Dent Oral Epidemiol 1989;17:7-10

5. Ohrbach R, Larsson P, List T. The jaw functional limitation scale: development, reliability, and validity of 8-item and 20-item versions. J Orofac Pain 2008;22:219-230.

6. Gotfredsen K, Walls AW. What dentition assures oral function? Clin Oral Implants Res 2007;18 Suppl 3:34-45.

7. Pereira LJ, Duarte Gaviao MB, Van Der Bilt A. Influence of oral characteristics and food products on masticatory function. Acta Odontol Scand 2006;64:193-201.

8. Peroz I, Tai S. Masticatory performance in patients with anterior disk displacement without reduction in comparison with symptom-free volunteers. Eur J Oral Sci 2002;110:341-344.

9. Kurita H, Ohtsuka A, Kurashina K, Kopp S. Chewing ability as a parameter for evaluating the disability of patients with temporomandibular disorders. J Oral Rehabil 2001;28:463-465.

10. Yap AU, Chua EK, Hoe JK. Clinical TMD, pain-related disability and psychological status of TMD patients. J Oral Rehabil 2002;29:374-380.

11. Felício CM, Melchior Mde O, Silva MA, Celeghini RM. [Masticatory performance in adults related to temporomandibular disorder and dental occlusion]. Pro Fono 2007;19:151-158. Portuguese.

12. Schiffman E, Ohrbach R, Truelove E, et al. Diagnostic Criteria for Temporomandibular Disorders (DC/TMD) for clinical and research 
applications: recommendations of the International RDC/TMD Consortium Network and Orofacial Pain Special Interest Group. J Oral Facial Pain Headache 2014;28:6-27.

13. Costen JB. A syndrome of ear and sinus symptoms dependent upon disturbed function of the temporomandibular joint. 1934. Ann Otol Rhinol Laryngol 1997;106(10 Pt 1):805-819.

14. Ohrbach R, Dworkin SF. The evolution of TMD diagnosis: past, present, future. J Dent Res 2016;95:1093-1101.

15. List T, Jensen RH. Temporomandibular disorders: Old ideas and new concepts. Cephalalgia 2017;37:692-704.

16. Slade GD, Ohrbach R, Greenspan JD, et al. Painful temporomandibular disorder: decade of discovery from OPPERA studies. $\mathrm{J}$ Dent Res 2016;95:1084-1092.

17. Epker J, Gatchel RJ. Coping profile differences in the biopsychosocial functioning of patients with temporomandibular disorder. Psychosom Med 2000;62:69-75.

18. LeResche L. Epidemiology of temporomandibular disorders: implications for the investigation of etiologic factors. Crit Rev Oral Biol Med 1997;8:291-305.

19. Sena MF, Mesquita KS, Santos FR, Silva FW, Serrano KV. Prevalence of temporomandibular dysfunction in children and adolescents. Rev Paul Pediatr 2013;31:538-545.

20. Gil-Martínez A, Paris-Alemany A, López-de-Uralde-Villanueva I, La Touche R. Management of pain in patients with temporomandibular disorder (TMD): challenges and solutions. J Pain Res 2018;11:571-587.

21. Maixner W, Diatchenko L, Dubner R, et al. Orofacial pain prospective evaluation and risk assessment study--the OPPERA study. J Pain 2011;12(11 Suppl):T4-T11.e1-e2.

22. Pereira LJ, Pereira-Cenci T, Pereira SM, et al. Psychological factors and the incidence of temporomandibular disorders in early adolescence. Braz Oral Res 2009;23:155-160.

23. Guarda-Nardini L, Pavan C, Arveda N, Ferronato G, Manfredini D. Psychometric features of temporomandibular disorders patients in relation to pain diffusion, location, intensity and duration. J Oral Rehabil 2012;39:737-743.

24. Song KW, Kim ME. Sleep quality of patients with temporomandibular disorders: relationship to clinical and psychological characteristics. J Oral Med Pain 2015;40:155-162.

25. Park JH, Kim HK, Kim KS, Kim ME. Pain catastrophizing for patients with temporomandibular disorders. J Oral Med Pain 2015;40:47-54

26. Choi SH, Kim KS, Kim ME. Pain disability of orofacial pain patients. J Oral Med Pain 2009;34:217-225.

27. Visscher CM, Ohrbach R, van Wijk AJ, Wilkosz M, Naeije M. The Tampa Scale for Kinesiophobia for temporomandibular disorders (TSK-TMD). Pain 2010;150:492-500.

28. LeResche L, Mancl LA, Drangsholt MT, Huang G, Von Korff M. Predictors of onset of facial pain and temporomandibular disorders in early adolescence. Pain 2007;129:269-278.

29. American Society of Anesthesiologists (ASA). ASA physical status classification system [Internet]. Schaumburg: ASA; c2019 [cited 2019 Jun 1]. Available from: https://www.asahq.org/standardsand-guidelines/asa-physical-status-classification-system.

30. International Network for Orofacial Pain and Related Disorders
Methodology (INFORM). Research Diagnostic Criteria for Temporomandibular Disorders (RDC/TMD) [Internet]. Buffalo: INFORM; c2019 [cited 2019 Jun 1]. Available from: https://ubwp. buffalo.edu/rdc-tmdinternational/tmd-assessmentdiagnosis/rdctmd/.

31. Ohrbach R, Granger C, List T, Dworkin S. Preliminary development and validation of the Jaw Functional Limitation Scale. Community Dent Oral Epidemiol 2008;36:228-236.

32. Zhang Y, Chung JW, Ohrbach R, Chung SC. Reliability of a korean version of the Jaw Functional Limitation Scale (JFLS) in dental college students. J Oral Med Pain 2004;29:197-203.

33. Cleeland CS. The brief pain inventory [Internet]. Texas: The University of Texas MD Anderson Cancer Center; c2019 [cited 2019 Jun 1]. Available from: https://www.mdanderson.org/research/ departments-labs-institutes/departments-divisions/symptomresearch/symptom-assessment-tools/brief-pain-inventory.html.

34. Stanhope J. Brief pain inventory review. Occup Med (Lond) 2016;66:496-497.

35. Yun YH, Mendoza TR, Heo DS, et al. Development of a cancer pain assessment tool in Korea: a validation study of a Korean version of the brief pain inventory. Oncology 2004;66:439-444.

36. Sullivan MJL, Bishop SR, Pivik J. The Pain Catastrophizing Scale: development and validation. Psychol Assess 1995;7:524-532.

37. Sullivan MJL. The Pain Catastrophizing Scale [Internet]. Montreal: Frederic Chappe; c2019 [cited 2019 Jun 1]. Available from: http://sullivan-painresearch.mcgill.ca/pcs.php.

38. Cho S, Kim HY, Lee JH. Validation of the Korean version of the Pain Catastrophizing Scale in patients with chronic non-cancer pain. Qual Life Res 2013;22:1767-1772.

39. Derogatis LR. Symptom checklist-90 revised (SCL-90-R) [Internet]. Clearwater: Statistics Solution; c2019 [cited 2019 Jun 1]. Available from: https://www.statisticssolutions.com/wp-content/ uploads/wp-post-to-pdf-enhanced-cache/1/symptom-checklist90-revised-scl-90-r.pdf.

40. Park IH, Jang JH, Chung JW. Reliability of the Korean version of tampa scale for kinesiophobia for temporomandibular disorders. J Oral Med Pain 2018;43:34-40.

41. Liu F, Steinkeler A. Epidemiology, diagnosis, and treatment of temporomandibular disorders. Dent Clin North Am 2013;57:465479.

42. Fernández-de-las-Peñas C, Galán-del-Río F, Fernández-Carnero J, Pesquera J, Arendt-Nielsen L, Svensson P. Bilateral widespread mechanical pain sensitivity in women with myofascial temporomandibular disorder: evidence of impairment in central nociceptive processing. J Pain 2009;10:1170-1178.

43. Graven-Nielsen T, Arendt-Nielsen L. Peripheral and central sensitization in musculoskeletal pain disorders: an experimental approach. Curr Rheumatol Rep 2002;4:313-321.

44. Healy GM, Finn DP, O'Gorman DA, et al. Pretreatment anxiety and pain acceptance are associated with response to trigger point injection therapy for chronic myofascial pain. Pain Med 2015;16:1955-1966.

45. Vlaeyen JW, Kole-Snijders AM, Boeren RG, van Eek H. Fear of movement/(re)injury in chronic low back pain and its relation to behavioral performance. Pain 1995;62:363-372. 
46. Gil-Martínez A, Grande-Alonso M, López-de-Uralde-Villanueva I, López-López A, Fernández-Carnero J, La Touche R. Chronic Temporomandibular Disorders: disability, pain intensity and fear of movement. J Headache Pain 2016;17:103.

47. Eccleston C. Role of psychology in pain management. Br J Anaesth 2001;87:144-152.

48. He S, Wang J, Ji P. Validation of the Tampa Scale for Kinesiopho- bia for Temporomandibular Disorders (TSK-TMD) in patients with painful TMD. J Headache Pain 2016;17:109.

49. Edlund J, Lamm CJ. Masticatory efficiency. J Oral Rehabil 1980; 7:123-130.

50. Ferreira MC, Porto de Toledo I, Dutra KL, et al. Association between chewing dysfunctions and temporomandibular disorders: a systematic review. J Oral Rehabil 2018;45:819-835. 